\title{
The impact of voluntary counselling and testing services on sexual behaviour change and HIV incidence: observations from a cohort study in rural Tanzania
}

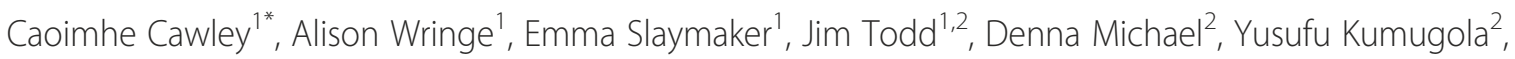
Mark Urassa ${ }^{2}$ and Basia Zaba ${ }^{1}$

\begin{abstract}
Background: It is widely assumed that voluntary counselling and testing $(V C T)$ services contribute to HIV prevention by motivating clients to reduce sexual risk-taking. However, findings from sub-Saharan Africa have been mixed, particularly among HIV-negative persons. We explored associations between VCT use and changes in sexual risk behaviours and HIV incidence using data from a community HIV cohort study in northwest Tanzania.

Methods: Data on VCT use, sexual behaviour and HIV status were available from three HIV serological surveillance rounds undertaken in 2003-4 (Sero4), 2006-7 (Sero5) and 2010 (Sero6). We used multinomial logistic regression to assess changes in sexual risk behaviours between rounds, and Poisson regression to estimate HIV incidence.

Results: The analyses included 3,613 participants attending Sero4 and Sero5 (3,474 HIV-negative and 139 HIV-positive at earlier round) and 2,998 attending Sero5 and Sero6 (2,858 HIV-negative and 140 HIV-positive at earlier round). Among HIV-negative individuals VCT use was associated with reductions in the number of sexual partners in the last year (aRR Seros 4-5: 1.42, 95\% Cl 1.07-1.88; aRR Seros 5-6: 1.68, 95\% Cl 1.25-2.26) and in the likelihood of having a non-cohabiting partner in the last year (aRR Seros 4-5: 1.57, 95\% Cl 1.10-2.25; aRR Seros 5-6: 1.48, 95\% Cl 1.07-2.04) or a high-risk partner in the last year (aRR Seros 5-6 1.57, 95\% Cl 1.06-2.31). However, VCT was also associated with stopping using condoms with non-cohabiting partners between Seros $4-5$ (aRR 4.88, 95\% Cl 1.39-17.16). There were no statistically significant associations between VCT use and changes in HIV incidence, nor changes in sexual behaviour among HIV-positive individuals, possibly due to small sample sizes.

Conclusions: We found moderate associations between VCT use and reductions in some sexual risk behaviours among HIV-negative participants, but no impacts among HIV-positive individuals in the context of low overall VCT uptake. Furthermore, there were no significant changes in HIV incidence associated with VCT use, although declining background incidence and small sample sizes may have prevented us from detecting this. The impact of VCT services will ultimately depend upon rates of uptake, with further research required to better understand processes of behaviour change following VCT use.
\end{abstract}

Keywords: Voluntary counselling and testing, HIV, Sexual behaviour, Tanzania, Cohort study

\footnotetext{
* Correspondence: caoimhe.cawley@lshtm.ac.uk

'Department of Population Health, London School of Hygiene and Tropical Medicine, Keppel Street, London WC1E 7HT, UK

Full list of author information is available at the end of the article
} 


\section{Background}

Voluntary counselling and testing (VCT) for HIV has been promoted as a gateway for access to HIV treatment and care. Concurrently, the services' potential contribution to HIV prevention has been emphasized; it is hypothesized that knowledge of HIV sero-status, accompanied by tailored and targeted risk reduction counselling, will assist both HIV-negative and HIV-positive individuals to reduce sexual risk-taking and protect themselves and their partners from HIV [1,2]. Previous studies have found mixed results with regard to the impact of VCT on reductions in sexual risk behaviour. These have generally found greater impact among individuals testing HIV-positive compared to those testing negative [3-5]. However, some studies have found no association between VCT use and changes in sexual behaviour [6,7], while others have found increases in sexual risk taking among individuals testing HIV-negative [8]. Few studies have assessed the impact of VCT on HIV incidence, however overall, these have found no significant differences between participants who received and did not receive VCT [5].

The measurement and comparison of indicators of sexual risk behaviour over time and between populations is difficult for a number of reasons, possibly explaining previous ambiguous findings regarding the impact of VCT on sexual behaviour change. Firstly, self-reported sexual behaviour data are often subject to recall and social desirability biases, the levels of which may vary depending on the behaviour in question, the amount of time that has passed since the event, and past exposure to HIV prevention messages [9]. Secondly, developing measures or indicators of sexual risk behaviour which are specific enough to accurately capture information on an individual's sexual risk profile, yet general enough to be comparable across populations or over time, is difficult [10]. Nevertheless, while measures of sexual behaviour have not always correlated well with rates of HIV incidence or prevalence [11-13], trends in these indicators have aided our understanding of the HIV epidemic and its relationship with sexual risk behaviour [14].

In this paper, we assess the impact of VCT use on changes in reported sexual risk behaviours and HIV incidence using data from consecutive rounds of HIV serological surveillance (sero-surveys) which have been carried out as part of an on-going community cohort study in northwest Tanzania since 1994. VCT services were provided during three sero-surveys in 2003-4, 2006-7 and 2010.

\section{Methods}

\section{Study setting}

The study setting in Mwanza region has been described in detail by Mwaluko et al. [15]. Briefly, the study area lies approximately $20 \mathrm{~km}$ to the east of Mwanza city and consists of six villages which make up the administrative ward of Kisesa with a combined population of approximately 32,000 people, roughly half of whom are adults. The cohort consists of rounds of demographic surveillance taking place approximately once every six months since 1994, and rounds of HIV serological surveillance among adults aged 15 or older once every two to three years. HIV prevalence in the study area rose from $6.0 \%$ (95\% CI $5.3 \%$ to $6.4 \%$ ) in $1994-5$ to $8.2 \%$ (95\% CI $7.7 \%$ to $8.8 \%$ ) in $2003-4$ [16], before falling to $6.5 \%$ (95\% CI $6.0 \%$ to $7.0 \%$ ) in 2010 (R. Isingo, personal communication). This pattern may be explained by net in-migration of HIV infected individuals into the study area in the 1990's, and a falling HIV incidence in the study area since the late 1990s [17].

Sero-surveys take place at a central point in each village, with dry-blood spot samples taken for ascertainment of HIV-status using a protocol based on informed consent without disclosure. HIV diagnosis is based on two ELIZAs (Uniform 2, Enzygnost HIV1/HIV2). A detailed questionnaire is administered by same sex interviewers, covering topics on socio-demographic characteristics, sexual behaviour and participants' previous use of VCT services including the place and circumstances of testing, e.g. whether at a previous sero-survey or elsewhere (and if so, where).

Since the fourth sero-survey round in 2003-4 (Sero4), a separate VCT service following Tanzanian national guidelines and including pre- and post-test counselling [18] has been available to all study participants. Individuals wishing to know their HIV status are directed to a purposeconstructed hut for VCT with a trained counsellor directly after completing their questionnaire interview. At Sero4, venous blood was collected and transported to the National Institute for Medical Research (NIMR) in Mwanza, with diagnosis based on two ELIZAs as for research tests, and clients asked to return for their test results and post-test counselling one week later. During the fifth and sixth serosurvey rounds in 2006-7 (Sero5) and 2010 (Sero6) venous blood was again collected but rapid HIV screening tests were used (preliminary test using Capillus, confirmatory test using Determine. Discrepant results resolved using two ELIZAs as for research tests). Quality control was performed at the NIMR laboratory on a 5\% sub-sample using two ELIZAs. During Sero5 and Sero6, HIV results and post-test counselling were usually available within $45 \mathrm{mi}$ nutes of the rapid test being performed. Data on the uptake of VCT at sero-surveys are anonymously linked to the main sero-survey questionnaire using unique numerical participant identifiers.

Although free antiretroviral therapy (ART) was not available in 2003-4, individuals with an HIV-positive result after using VCT at Sero4 were informed that treatment would become available in the region in the near future through the national ART programme, which started 
at the beginning of 2005. With their prior agreement, these individuals were subsequently traced by the VCT counsellors and referred to the zonal referral hospital in Mwanza city for follow-up care. Individuals using VCT and testing HIV-positive at Sero5 were referred directly to Mwanza city hospitals. By Sero6 in 2010, a care and treatment centre was available locally at the health centre in Kisesa and so HIV-positive patients were referred here.

\section{Other health services in the study area}

The study population is served by a government-run health centre located in Kisesa trading centre, by three small government-run dispensaries located in the rural villages, and by a number of private clinics located mainly in the trading centre. A stand-alone VCT clinic has been available at Kisesa health centre since 2005, while provider initiated testing and counselling (PITC) has been offered to all pregnant women attending the health centre for antenatal care since the end of 2008 . PITC has also been available at the out-patients department since 2010, where testing may be offered to patients attending the sexually transmitted infections or tuberculosis clinics. Since mid-2009, antenatal PITC is sometimes offered to women attending the small rural dispensaries, dependant on the availability of test-kit supplies.

\section{Ethical statement}

Ethical approval for the Kisesa cohort study has been granted by the Tanzanian Medical Research Coordinating Committee and the Ethics Committee of the London School of Hygiene and Tropical Medicine. Participation is based on informed consent without disclosure of HIVresearch test results, however since Sero4 (just prior to the introduction of ART), participants are additionally offered a free VCT service as detailed above. During Sero4 verbal consent was obtained, due to low literacy rates among the study population. This was witnessed and documented for each participant on their study questionnaire, by a member of the sero-survey team. During Sero5 and Sero6 written consent was introduced (either a signature or a thumb-print, depending on the participant's writing ability).

\section{Data and analysis}

Nine indices of sexual behaviour were used: the number of sex partners in the last year, the acquisition or loss of spouse, regular non-cohabiting sexual partner, or high risk sex partner in the last year, whether a condom was used with spouse, regular non-cohabiting sexual partner, or high risk partner at last sex, and the coital frequency with spouse or regular non-cohabiting sexual partner in the last week. Changes in sexual behaviour were determined by comparing behaviours reported at one round with those reported at the next, in order to determine whether there had been an increase, decrease or no change in behaviour. For quantitative variables (number of sexual partners, coital frequency), differences in the constructed behaviour change variables greater than zero indicated an increase in sexual risk behaviour while values less than zero indicated a decrease and a value of zero indicated no change. For changes in binary variables (condom use at last sex), the indices represented the direction of change in behaviour, with 1 representing increased risk, 0 no change and -1 decreased risk. Multinomial logistic regression was used to assess the association between VCT and each of the nine indices of sexual behaviour change. Crude associations were adjusted for socio-demographic variables (age, sex, marital status, area of residence, level of education and reported previous use of VCT) in multivariable analyses.

In order to explore any potential differences over time, analyses were carried out separately for those a) attending both Sero4 and Sero5 and using the VCT service at Sero4, b) attending both Sero5 and Sero6 and using the VCT service at Sero5. Analyses were restricted to the non-virgin population (those who reported having ever had sex) and considered separately for individuals testing HIV-negative and HIV-positive at the time of going for VCT.

HIV incidence was estimated based on the number of initially HIV-negative respondents who seroconverted between Seros 4 and 5, and Seros 5 and 6, respectively. Dates of sero-conversion were assigned based on the midpoint between sero-survey interview dates. Poisson regression models were used to calculate crude and adjusted HIV incidence rates comparing VCT users and non-users.

\section{Results}

\section{Descriptive characteristics}

In total 8,961 participants attended Sero4 (participation rate of $66 \%$ [16]), of whom 3,923 (43.8\%) also attended Sero5. Of these, 3,613/3,923 (92.1\%) reported that they had ever had sex. 933/3,613 (25.8\%) individuals expressed a desire for VCT while 324/3,613 (9.0\%) actually returned to receive their results and complete VCT. In total 8,696 participants attended Sero5 (participation rate of 61\% [17]), of whom 3,509 also attended Sero6 (40.3\%), with $2,998 / 3,509$ (85.4\%) reporting having ever had sex. Of these, $525 / 2,998$ (17.5\%) individuals expressed a desire for VCT while 532/2,998 (17.7\%) actually completed VCT. The descriptive characteristics of the non-virgin population attending the two sets of sero-survey rounds are shown in Table 1. The largest proportions of attendees were female and fell in the $\geq 45$ age-group. Of those attending Sero4 and Sero5, 139/3,613 participants (3.8\%) were HIV-positive, while $23 / 324$ (7.1\%) of those who used VCT at Sero4 were HIV-positive. Of those attending Sero5 and Sero6, 140/ 2,998 (4.7\%) were HIV-positive and 29/532 (5.4\%) of those who used VCT at Sero5 were HIV-positive. 
Table 1 Characteristics (at earlier round) of individuals attending i)Sero-4 and Sero-5, ii)Sero-5 and Sero-6, by VCT use at earlier round

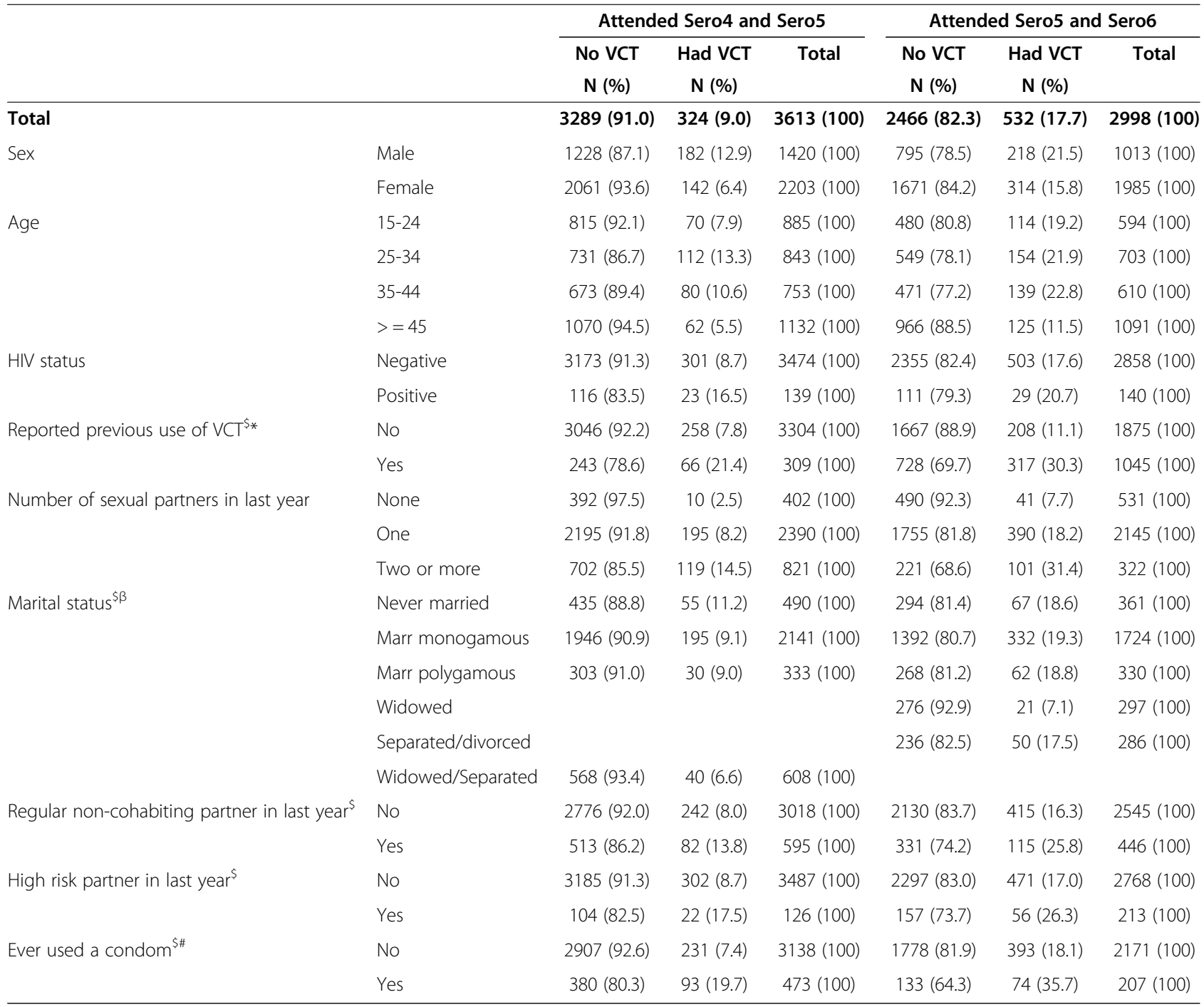

${ }^{\$}$ Small numbers of missing data $(<3 \%)$.

*This was previous VCT use as reported at the later round (to account for any VCT use in the inter sero-survey period).

${ }^{\beta}$ Data on widowed/separated not broken down at Sero4.

${ }^{\#}$ At Sero5 this variable related to ever use of condoms with spouse or regular non-cohabiting partners only $(N=2,403)$.

Across both sets of rounds, approximately $60 \%$ of attendees were married monogamously with a further $10 \%$ being in polygamous marriages. Most participants reported having one sexual partner (in the last year), and not having any regular non-cohabiting or high risk partners (also in the last year - Table 1). Condom use was rare, with just 7.4\% reporting ever using a condom at Sero4, and 9.2\% reporting ever using a condom with the three most recent sexual partners at Sero5.

\section{Impact of VCT on sexual behaviour change among} HIV-negative individuals

Among HIV-negative individuals, VCT use was associated with some changes in sexual behaviour in terms of the number and type of partnerships formed, but less so in terms of condom use or coital frequency (see Tables 2 and 3). Between Sero4 and Sero5, compared to those who did not have VCT, VCT users had an increased likelihood of reducing their number of sexual partners in the last year (Table 2: adjusted risk ratio [aRR] 1.42, 95\% confidence interval [CI] 1.07-1.88). A similar pattern was seen in terms of reduction in the number of sexual partners between Sero5 and Sero6 (Table 3: aRR 1.68, 95\% CI 1.25-2.26).

Among HIV negative individuals, there was evidence that VCT use at Sero4 was associated with both acquiring (aRR 1.88, 95\% CI 1.29-2.74) and losing (aRR 1.56, 95\% CI 0.95-2.55) a spouse by Sero5 (Table 2). While 
Table 2 Change in sexual behaviour between Sero4 and Sero5 associated with VCT use at Sero4, HIV-negative individuals

\begin{tabular}{|c|c|c|c|c|c|c|}
\hline & \multicolumn{4}{|c|}{$\mathrm{N}$ with outcome (\%) } & \multirow[b]{2}{*}{ Crude risk ratio $(95 \% \mathrm{Cl})$} & \multirow[b]{2}{*}{ Adjusted risk ratio $(95 \% \mathrm{Cl})^{9}$} \\
\hline & \multicolumn{2}{|c|}{ No VCT } & \multicolumn{2}{|c|}{ Had VCT } & & \\
\hline \multicolumn{7}{|c|}{ Number of sex partners in last year } \\
\hline & 3173 & $\%$ & 301 & $\%$ & & \\
\hline No change & 2060 & 64.9 & 169 & 56.1 & 1 & \\
\hline Increase & 306 & 9.6 & 25 & 8.3 & $1(0.64-1.54)$ & $0.82(0.52-1.30)$ \\
\hline Decrease & 807 & 25.4 & 107 & 35.5 & $1.62(1.25-2.09)^{* * *}$ & $1.42(1.07-1.88)^{* *}$ \\
\hline \multicolumn{7}{|c|}{ Acquired or lost a spouse ${ }^{a}$} \\
\hline & 3136 & $\%$ & 297 & $\%$ & & \\
\hline No change & 2701 & 86.1 & 233 & 78.5 & 1 & 1 \\
\hline Acquired & 255 & 8.1 & 43 & 14.5 & $1.95(1.38-2.77)^{* * *}$ & $1.88(1.29-2.74)^{* * *}$ \\
\hline Lost & 180 & 5.7 & 21 & 7.1 & $1.35(0.84-2.17)$ & $1.56(0.95-2.55)^{*}$ \\
\hline \multicolumn{7}{|c|}{ Acquired or lost a regular non-cohabiting partner } \\
\hline & 3168 & $\%$ & 301 & $\%$ & & \\
\hline No change & 2601 & 82.1 & 226 & 75.1 & 1 & 1 \\
\hline Acquired & 251 & 7.9 & 27 & 9.0 & $1.24(0.81-1.88)$ & $0.88(0.55-1.43)$ \\
\hline Lost & 316 & 10.0 & 48 & 15.9 & $1.75(1.25-2.44)^{* * *}$ & $1.57(1.10-2.25)^{* *}$ \\
\hline \multicolumn{7}{|c|}{ Acquired or lost a high risk partner } \\
\hline & 3156 & $\%$ & 299 & $\%$ & & \\
\hline No change & 2879 & 91.2 & 267 & 89.3 & 1 & 1 \\
\hline Acquired & 193 & 6.1 & 19 & 6.4 & $1.06(0.65-1.73)$ & $0.97(0.58-1.62)$ \\
\hline Lost & 84 & 2.7 & 13 & 4.3 & $1.67(0.92-3.03)$ & $1.41(0.75-2.65)$ \\
\hline \multicolumn{7}{|c|}{ Condom use at last sex with spouse $\mathrm{e}^{\#}$} \\
\hline & 2000 & $\%$ & 190 & $\%$ & & \\
\hline No change & 1951 & 97.6 & 184 & 96.8 & 1 & 1 \\
\hline Stopped using & 31 & 1.6 & 2 & 1.1 & $0.68(0.16-2.88)$ & $0.45(0.10-1.93)$ \\
\hline Started using & 18 & 0.9 & 4 & 2.1 & $2.36(0.79-7.04)$ & $1.79(0.56-5.67)$ \\
\hline \multicolumn{7}{|c|}{ Condom use at last sex with regular non-cohabiting partner ${ }^{\#}$} \\
\hline & 179 & $\%$ & 31 & $\%$ & & \\
\hline No change & 147 & 82.1 & 17 & 54.8 & 1 & 1 \\
\hline Stopped using & 12 & 6.7 & 8 & 25.8 & $5.76(2.07-16.08)^{* * *}$ & $4.88(1.39-17.16)^{* *}$ \\
\hline Started using & 20 & 11.2 & 6 & 19.4 & $2.59(0.92-7.35)$ & $2.21(0.60-8.14)$ \\
\hline \multicolumn{7}{|c|}{ Condom use at last sex with high risk partner ${ }^{\#}$} \\
\hline & 12 & $\%$ & 5 & $\%$ & & \\
\hline No change & 8 & 66.7 & 3 & 60.0 & 1 & - \\
\hline Stopped using & 1 & 8.3 & 1 & 20.0 & $2.67(0.12-57.62)$ & - \\
\hline Started using & 3 & 25.0 & 1 & 20.0 & $0.89(0.06-12.25)$ & - \\
\hline
\end{tabular}

"All outcomes adjusted for age, sex, marital status, area of residence, level of education and previous VCT use as reported at the later sero-survey round. *p $\leq 0.1, * * p \leq 0.05, * * * p \leq 0.001$.

a'Acquired or lost spouse' was not adjusted for marital status (as this was the outcome).

${ }^{\#}$ Analyses conduced among those with relevant partner type at both rounds only.

similar trends were seen between Sero5 and Sero6, the results were not statistically significant (Table 3 aRR for acquiring a spouse: $1.19,95 \%$ CI $0.82-1.73$; aRR for losing a spouse: 1.12, 95\% CI 0.74-1.70). Between Seros 4 and 5 and also between Seros 5 and 6, VCT use at the earlier round was also significantly associated with losing a regular non-cohabiting partner by the later round (aRR Seros 4-5: 1.57, 95\% CI 1.10-2.25, aRR Seros 5-6: 1.48, 95\% CI 1.07-2.04). Further investigation revealed that approximately half of those who acquired a spouse 
Table 3 Change in sexual behaviour between Sero5 and Sero6 associated with VCT use at Sero5, HIV-negative individuals

\begin{tabular}{|c|c|c|c|c|c|c|}
\hline & \multicolumn{4}{|c|}{$\mathrm{N}$ with outcome (\%) } & \multirow[b]{2}{*}{ Crude risk ratio $(95 \% \mathrm{Cl})$} & \multirow[b]{2}{*}{ Adjusted risk ratio $(95 \% \mathrm{Cl})$} \\
\hline & \multicolumn{2}{|c|}{ No VCT } & \multicolumn{2}{|c|}{ Had VCT } & & \\
\hline \multicolumn{7}{|c|}{ Number of sex partners in last year } \\
\hline & 2336 & $\%$ & 502 & $\%$ & & \\
\hline No change & 1732 & 74.1 & 338 & 67.3 & 1 & 1 \\
\hline Increase & 272 & 11.6 & 74 & 14.7 & $1.39(1.05,1.85)^{* *}$ & $1.13(0.82,1.55)$ \\
\hline Decrease & 332 & 14.2 & 90 & 17.9 & $1.39(1.07,1.80)^{* *}$ & $1.68(1.25,2.26)^{* * *}$ \\
\hline \multicolumn{7}{|c|}{ Acquired or lost a spouse $^{a}$} \\
\hline & 2346 & $\%$ & 500 & $\%$ & & \\
\hline No change & 2043 & 87.1 & 423 & 84.6 & 1 & 1 \\
\hline Acquired & 158 & 6.7 & 44 & 8.8 & $1.35(0.95,1.91)^{*}$ & $1.19(0.82,1.73)$ \\
\hline Lost & 145 & 6.2 & 33 & 6.6 & $1.1(0.74,1.63)$ & $1.12(0.74,1.70)$ \\
\hline
\end{tabular}

Acquired or lost a regular non-cohabiting partner

$\begin{array}{lcccccc} & \mathbf{2 3 5 0} & \mathbf{\%} & \mathbf{5 0 1} & \mathbf{\%} & & 1 \\ \text { No change } & 2017 & 85.8 & 399 & 79.6 & 1 & 1.23(0.73,2.07) \\ \text { Acquired } & 90 & 3.8 & 23 & 4.6 & 1.29(0.81,2.07) & 1.48(1.07,2.04)^{* *} \\ \text { Lost } & 243 & 10.3 & 79 & 15.8 & 1.64(1.25,2.16)^{* *} & \end{array}$

Acquired or lost a high risk or casual partner ${ }^{\Omega}$

$\begin{array}{lccc} & \mathbf{2 3 4 4} & \mathbf{\%} & \mathbf{4 9 8} \\ \text { No change } & 2062 & 88.0 & 406 \\ \text { Acquired } & 153 & 6.5 & 48 \\ \text { Lost } & 129 & 5.5 & 44\end{array}$

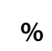

$$
81.5
$$$$
9.6
$$$$
8.8
$$

Condom use at last sex with spouse ${ }^{\#}$

$\begin{array}{lccc} & \mathbf{1 3 7 9} & \mathbf{\%} & \mathbf{3 3 2} \\ \text { No change } & 1345 & 97.5 & 326 \\ \text { Stopped using } & 18 & 1.3 & 3 \\ \text { Started using } & 16 & 1.2 & 3\end{array}$

Condom use at last sex with regular non-cohabiting partner

$\begin{array}{llcc} & \mathbf{7 5} & \mathbf{\%} & \mathbf{2 9} \\ \text { No change } & 51 & 68.0 & 20 \\ \text { Stopped using } & 12 & 16.0 & 5 \\ \text { Started using } & 12 & 16.0 & 4\end{array}$

Condom use at last sex with high risk or casual partner $\# \Omega$

$\begin{array}{lccccl} & \mathbf{1 8} & \% & \mathbf{1 0} & \% & \\ \text { No change } & 10 & 55.6 & 6 & 60.0 & 1 \\ \text { Stopped using } & 1 & 5.6 & 2 & 20.0 & 3.33(0.25,45.11) \\ \text { Started using } & 7 & 38.9 & 2 & 20.0 & 0.48(0.07,3.09)\end{array}$

Number times sex with spouse in last week ${ }^{\#}$

$\begin{array}{lccc} & \mathbf{1 3 8 8} & \% & \mathbf{3 3 5} \\ \text { No change } & 384 & 27.7 & 81 \\ \text { Increase } & 296 & 21.3 & 68 \\ \text { Decrease } & 708 & 51.0 & 186\end{array}$

20.3

55.5
$1.28(0.84,1.95)$

$1.57(1.06,2.31)^{* *}$
$\%$

98.2

0.9

0.9

$\%$

69.0

17.2

$1.06(0.33,3.40)$

$0.85(0.24,2.95)$

$0.70(0.14,3.54)$

$0.32(0.06,1.63)$
$0.64(0.18,2.28)$

$0.62(0.17,2.29)$ 
Table 3 Change in sexual behaviour between Sero5 and Sero6 associated with VCT use at Sero5, HIV-negative individuals (Continued)

\begin{tabular}{|c|c|c|c|c|c|c|}
\hline \multicolumn{7}{|c|}{ Number times sex with regular non-cohabiting partner in last week" } \\
\hline & 75 & $\%$ & 29 & $\%$ & & \\
\hline No change & 27 & 36.0 & 11 & 37.9 & 1 & 1 \\
\hline Increase & 19 & 25.3 & 7 & 24.1 & $0.9(0.30,2.76)$ & $0.83(0.21,3.32)$ \\
\hline Decrease & 29 & 38.7 & 11 & 37.9 & $0.93(0.35,2.50)$ & $0.92(0.27,3.14)$ \\
\hline
\end{tabular}

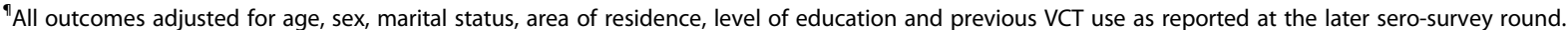
${ }^{*} p \leq 0.1,{ }^{* *} p \leq 0.05,{ }^{* * *} p \leq 0.001$.

a'Acquired or lost spouse' was not adjusted for marital status (as this was the outcome).

${ }^{\Omega}$ Questions related to 'casual' rather than 'high risk' partners at Sero6, however, the two categories were considered comparable.

\#Analyses conduced among those with relevant partner type at both rounds only.

by the later round were the same people who had lost a regular non-cohabiting partner $(144 / 298$ or $48.3 \%$ of those acquiring a spouse between Sero4 and Sero5, and $106 / 202$ or $52.5 \%$ of those acquiring a spouse between Sero5 and Sero6). As a result, at least some of the spousal acquisition between rounds is likely as a result of people marrying their regular partners. Between Seros 5 and 6, VCT use was also associated with losing a highrisk or casual partner (aRR 1.57, 95\% CI 1.06-2.31).

In general there was no significant impact of VCT on condom use behaviour, although VCT at Sero4 was associated with an increased likelihood of stopping using condoms with regular non-cohabiting partners by Sero 5 (aRR 4.88, 95\% CI 1.39-17.16). Coital frequency information was not available at Sero4, but between Sero5 and Sero6, there was no evidence for an impact of VCT on changes in coital frequency with spouses or regular noncohabiting partners in the last week (Table 3). In addition, there was no evidence to suggest any interaction between VCT use and gender in terms of any of the sexual behaviour change outcomes.

In total 2,010 participants attended all three serosurveys, of whom 1,876 (93.3\%) reported having ever had sex. Among these, 82/1,876 (4.4\%) used VCT twice (at both Sero4 and Sero5) and 1,828/1,876 (97.4\%) were HIV-negative at Sero4. In crude analyses among HIV negative individuals, those using VCT twice (at Sero4 and Sero5) were more likely to report increasing their number of sexual partners (RR 2.10, 95\% CI 1.06-4.14) and acquiring a high-risk partner (RR 2.13, 95\% CI 1.034.38) between Sero4 and Sero6, compared to those who did not use VCT twice. However there were no significant impacts of VCT on any of the sexual behaviour change outcomes in adjusted analyses.

\section{Impact of VCT on HIV incidence}

Between Sero4 and Sero5, HIV incidence among those who did not use VCT at Sero4 was 0.86 per 100 person years (95\% CI $0.70-1.06)$, while it was 1.04 per 100 person years (95\% CI 0.56-1.94) among those who used VCT. The corresponding incidence between Sero5 and
Sero6 was 0.66 per 100 person years (95\% CI 0.46-0.93) among those who did not use VCT at Sero5 and 0.48 per 100 person years (95\% CI $0.20-1.16)$ among those used VCT. In crude and adjusted analyses, VCT was not significantly associated with changes in HIV-incidence between either set of sero-survey rounds (Table 4).

\section{Impact of VCT on sexual behaviour change among HIV-positive individuals}

Fewer indicators of sexual behaviour change could be assessed among HIV-positive individuals due to small sample sizes, and VCT use was not significantly associated with changes in any of these in crude or adjusted analyses (Tables 5 and 6).

However, for some indicators, the measures of effect seemed to go in opposite directions between Seros 4 and 5 and Seros 5 and 6. For example, a larger proportion of HIV-positive individuals using VCT at Sero4 lost a spouse by Sero5 (13.0\% versus $11.4 \%$, aRR 2.65 , 95\% CI 0.46-15.23) and acquired a regular non-cohabiting partner by Sero5 (30.4\% versus $17.5 \%$, aRR 3.46, 95\% CI $0.71-16.83)$ compared to those not using VCT. The opposite effect was seen between Seros 5 and 6. Compared to those not using VCT, HIV-positive individuals using VCT at Sero5 were more likely to acquire a spouse (10.3\% versus $2.7 \%$, aRR $4.35,95 \%$ CI $0.68-27.93$ ) and to lose a non-cohabiting partner (20.7\% versus $11.7 \%$, aRR 1.59 , 95\% CI 0.45-5.64) by Sero6. However, the confidence intervals around all of these estimates are very wide.

In crude analyses, VCT use at both Sero4 and Sero5 seemed to be associated with an increased likelihood of both stopping and starting using condoms at last sex with spouse by the next round (Tables 5 and 6). However, the numbers contributing to these analyses were very small (six participants between Seros 4 and 5, eight participants between Seros 5 and 6). As a result, the results were not statistically significant, nor was it possible to adjust for or investigate the effect of possible confounders.

Pooling the data for both sets of rounds (taking account of clustering for those who attended all three rounds) did reveal some evidence for an association 
Table 4 HIV incidence among initially HIV-negative participants, by VCT use at the earlier sero-survey round

\begin{tabular}{|c|c|c|c|c|c|c|}
\hline & $\mathbf{N}$ & Person years & Seroconversions & Incidence per 100PY & Crude rate ratio $(95 \% \mathrm{Cl})$ & Adjusted rate ratio $(95 \% \mathrm{Cl})^{*}$ \\
\hline \multicolumn{7}{|c|}{ Attended Sero4 and Sero5 } \\
\hline No VCT & 3160 & 10133.8 & 87 & $0.86(0.70-1.06)$ & 1 & 1 \\
\hline VCT & 299 & 959.1 & 10 & $1.04(0.56-1.94)$ & $1.21(0.63-2.34)$ & $1.13(0.58-2.22)$ \\
\hline \multicolumn{7}{|c|}{ Attended Sero5 and Sero6 } \\
\hline No VCT & 2343 & 4880.1 & 32 & $0.66(0.46-0.93)$ & 1 & 1 \\
\hline VCT & 501 & 1031.0 & 5 & $0.48(0.20-1.16)$ & $0.74(0.29-1.90)$ & $0.69(0.26-1.82)$ \\
\hline
\end{tabular}

*Adjusted for age, sex and previous VCT use as at reported at the later sero-survey round.

between VCT use and a reduction in some sexual risk behaviours between rounds (results not shown). In these analyses, HIV-positive individuals using VCT at the earlier round seemed less likely to increase their number of sexual partners in the last year by the next round as compared to those not using VCT (aRR 0.22, 95\% CI 0.05-1.01, $\mathrm{p}=0.05$ ).

\section{Discussion}

In this study, the overall uptake of VCT at the first two sero-surveys during which it was offered was low $(10 \%$ and $17 \%$ at Seros 4 and 5 respectively [19]). A recent estimate of ART coverage among those in need in Kisesa (based on individuals HIV-positive at Sero5) was also low (just $2 \%-[20])$. As in previous studies, our findings

Table 5 Change in sexual behaviour between Sero4 and Sero5 associated with VCT use at Sero4, HIV-positive individuals

\begin{tabular}{|c|c|c|c|c|c|c|}
\hline & \multicolumn{4}{|c|}{$\mathrm{N}$ with outcome (\%) } & \multirow[b]{2}{*}{ Crude risk ratio $(95 \% \mathrm{Cl})$} & \multirow[b]{2}{*}{ Adjusted risk ratio $(95 \% \mathrm{Cl})$} \\
\hline & \multicolumn{2}{|c|}{ No VCT } & \multicolumn{2}{|c|}{ Had VCT } & & \\
\hline \multicolumn{7}{|c|}{ Number of sex partners in last year } \\
\hline & 115 & $\%$ & 23 & $\%$ & & \\
\hline No change & 64 & 55.7 & 13 & 56.5 & 1 & 1 \\
\hline Increase & 16 & 13.9 & 0 & 0.0 & - & - \\
\hline Decrease & 35 & 30.4 & 10 & 43.5 & $1.41(0.56,3.54)$ & $0.88(0.25,3.02)$ \\
\hline \multicolumn{7}{|c|}{ Acquired or lost a spouse ${ }^{a}$} \\
\hline & 114 & $\%$ & 23 & $\%$ & & \\
\hline No change & 93 & 81.6 & 19 & 82.6 & 1 & 1 \\
\hline Acquired & 8 & 7.0 & 1 & 4.3 & $0.61(0.07,5.18)$ & $0.64(0.06,7.13)$ \\
\hline Lost & 13 & 11.4 & 3 & 13.0 & $1.13(0.29,4.35)$ & $2.65(0.46,15.23)$ \\
\hline \multicolumn{7}{|c|}{ Acquired or lost a regular non-cohabiting partner } \\
\hline & 114 & $\%$ & 23 & $\%$ & & \\
\hline No change & 79 & 69.3 & 15 & 65.2 & 1 & 1 \\
\hline Acquired & 20 & 17.5 & 7 & 30.4 & $1.84(0.66,5.12)$ & $3.46(0.71,16.83)$ \\
\hline Lost & 15 & 13.2 & 1 & 4.3 & $0.35(0.04,2.86)$ & $0.26(0.02,2.85)$ \\
\hline \multicolumn{7}{|c|}{ Acquired or lost a high risk partner } \\
\hline & 115 & $\%$ & 22 & $\%$ & & \\
\hline No change & 98 & 85.2 & 17 & 77.3 & 1 & 1 \\
\hline Acquired & 10 & 8.7 & 1 & 4.5 & $0.58(0.07,4.80)$ & $0.63(0.05,8.02)$ \\
\hline Lost & 7 & 6.1 & 4 & 18.2 & $3.29(0.87,12.48)$ & $3.10(0.50,19.31)$ \\
\hline \multicolumn{7}{|c|}{ Condom use at last sex with spouse ${ }^{\#}$} \\
\hline & 68 & $\%$ & 12 & $\%$ & & \\
\hline No change & 64 & 94.1 & 10 & 83.3 & 1 & 1 \\
\hline Stopped using & 2 & 2.9 & 1 & 8.3 & $3.2(0.26,38.64)$ & $0.99(0.03,34.27)$ \\
\hline Started using & 2 & 2.9 & 1 & 8.3 & $3.2(0.26,38.64)$ & - \\
\hline
\end{tabular}

"All outcomes adjusted for age, sex, marital status, area of residence, level of education and previous VCT use as reported at the later sero-survey round. a'Acquired or lost spouse' was not adjusted for marital status (as this was the outcome).

"Analyses conducted among those with a spouse at both rounds only. 
Table 6 Change in sexual behaviour between Sero5 and Sero6 associated with VCT use at the Sero5, HIV-positive individuals

\begin{tabular}{|c|c|c|c|c|c|c|}
\hline & \multicolumn{4}{|c|}{$\mathrm{N}$ with outcome (\%) } & \multirow[b]{2}{*}{ Crude risk ratio $(95 \% \mathrm{Cl})$} & \multirow[b]{2}{*}{ Adjusted risk ratio $(95 \% \mathrm{Cl})$} \\
\hline & \multicolumn{2}{|c|}{ No VCT } & \multicolumn{2}{|c|}{ Had VCT } & & \\
\hline \multicolumn{7}{|c|}{ Number of sex partners in last year } \\
\hline & 110 & $\%$ & 29 & $\%$ & & \\
\hline No change & 72 & 65.5 & 21 & 72.4 & 1 & 1 \\
\hline Increase & 14 & 12.7 & 3 & 10.3 & $0.73(0.19,2.80)$ & $0.41(0.08,2.12)$ \\
\hline Decrease & 24 & 21.8 & 5 & 17.2 & $0.71(0.24,2.10)$ & $0.56(0.13,2.38)$ \\
\hline \multicolumn{7}{|c|}{ Acquired or lost a spouse ${ }^{a}$} \\
\hline & 111 & $\%$ & 29 & $\%$ & & \\
\hline No change & 88 & 79.3 & 23 & 79.3 & 1 & 1 \\
\hline Acquired & 3 & 2.7 & 3 & 10.3 & $3.83(0.72,20.22)$ & $4.35(0.68,27.93)$ \\
\hline Lost & 20 & 18.0 & 3 & 10.3 & $0.57(0.16,2.10)$ & $0.68(0.17,2.63)$ \\
\hline \multicolumn{7}{|c|}{ Acquired or lost a regular non-cohabiting partner } \\
\hline & 111 & $\%$ & 29 & $\%$ & & \\
\hline No change & 90 & 81.1 & 22 & 75.9 & 1 & 1 \\
\hline Acquired & 8 & 7.2 & 1 & 3.4 & $0.51(0.06,4.31)$ & $0.21(0.01,3.25)$ \\
\hline Lost & 13 & 11.7 & 6 & 20.7 & $1.89(0.65,5.53)$ & $1.59(0.45,5.63)$ \\
\hline \multicolumn{7}{|c|}{ Acquired or lost a high risk or casual partner ${ }^{\Omega}$} \\
\hline & 110 & $\%$ & 29 & $\%$ & & \\
\hline No change & 90 & 81.8 & 25 & 86.2 & 1 & 1 \\
\hline Acquired & 11 & 10.0 & 3 & 10.3 & $0.98(0.25,3.79)$ & $0.53(0.10,2.98)$ \\
\hline Lost & 9 & 8.2 & 1 & 3.4 & $0.4(0.05,3.31)$ & $0.32(0.02,4.35)$ \\
\hline \multicolumn{7}{|c|}{ Condom use at last sex with spouse $\mathrm{H}^{\#}$} \\
\hline & 61 & $\%$ & 18 & $\%$ & & \\
\hline No change & 56 & 91.8 & 15 & 83.3 & 1 & 1 \\
\hline Stopped using & 2 & 3.3 & 1 & 5.6 & $1.87(0.16,22.01)$ & - \\
\hline Started using & 3 & 4.9 & 2 & 11.1 & $2.49(0.38,16.27)$ & - \\
\hline \multicolumn{7}{|c|}{ Number of times sex with spouse in last week ${ }^{\#}$} \\
\hline & 62 & $\%$ & 19 & $\%$ & & \\
\hline No change & 18 & 29.0 & 2 & 10.5 & 1 & 1 \\
\hline Increase & 14 & 22.6 & 3 & 15.8 & $1.93(0.28,13.16)$ & $3.37(0.26,44.11)$ \\
\hline Decrease & 30 & 48.4 & 14 & 73.7 & $4.2(0.85,20.65)$ & $7.55(0.84,67.63)$ \\
\hline
\end{tabular}

"All outcomes adjusted for age, sex, marital status, area of residence, level of education and previous VCT use as reported at the later sero-survey round.

'Acquired or lost spouse' was not adjusted for marital status (as this was the outcome).

${ }^{\Omega}$ Questions related to 'casual' rather than 'high risk' partners at Sero6, however, the two categories were considered comparable.

\#Analyses conducted among those with a spouse at both rounds only.

with regard to the impact of VCT on sexual behaviour change were mixed, with service use associated with changes in some behaviours but not others. However, it was encouraging that reductions in the numbers and types of partnerships formed persisted across both sets of sero-survey rounds.

We found no evidence for an impact of VCT on changes in HIV incidence. While it is possible that a declining incidence in the study area overall and a small number of sero-conversions may have prevented us from detecting this, a number of other studies have similarly found no impact of VCT on changes in HIV incidence $[6,21]$.

It was perhaps unsurprising that HIV-negative individuals were more likely to acquire a spouse after going for $\mathrm{VCT}$, as young couples in sub-Saharan African are frequently advised to go for VCT before marriage. The interpretation or significance of the acquisition or loss of a spouse is somewhat different to the acquisition or loss of a high-risk partner. While acquisition of the latter may clearly be interpreted as a transition to higher risk behaviour, the acquisition of a spouse may be viewed as a shift to 
lower-risk behaviour as people theoretically move from a 'sexually active but single' state to a more stable, settled relationship. Conversely, the loss of a spouse (which might not be volitional, for example if it occurred as a result of widowhood) could be viewed as a transition to higher-risk behaviour as people leave settled relationships and return to a phase during which they may seek out new sexual partners.

It was somewhat more surprising that HIV-negative individuals using VCT at Sero4 were more likely to lose a spouse by Sero5 than those not using VCT. However, of the $201 \mathrm{HIV}$-negative individuals who lost a spouse between Sero4 and Sero5, 75 of these cases (37.3\%) were as a result of widowhood. The observed association between VCT use and spousal loss between Seros 4-5 may be linked to widowhood through reverse causality, as individuals with sick partners may have been more likely to go and test than individuals with healthy partners. Further qualitative research may help to elucidate the reasons why people go (or don't go) for VCT, the types of information that clients receive and the perceived importance of this information in helping people to change their sexual behaviour.

Condom use among study participants was low overall and VCT use was generally associated with little change in this behaviour. The finding that VCT use at Sero4 was associated with an increased likelihood of stopping using condoms at last sex with regular non-cohabiting partners by Sero5 is difficult to interpret, but may relate to the specific risk-profile of these non-cohabiting partners and/or whether they had also been for VCT and tested HIV-negative. A number of studies have found VCT use to be associated with increases in condom use $[3,4,22]$ and so it was disappointing that we did not find similar results in this study. Condom use has previously been reported to be low in this part of Tanzania $[23,24]$, and it is possible that negative attitudes towards their use persist in spite of messages shared during VCT. Low levels of condom use could also relate to anecdotal evidence of problems relating to their availability or supply.

There were no statistically significant changes in sexual behaviour associated with VCT use among HIV-positive individuals, except for a reduced likelihood of increasing the number of sexual partners in the last year in the pooled analysis. In the analyses done separately by round, for some indicators the measures of effect seemed to go in opposite directions between the two sets of rounds. For example, between Seros 4-5, individuals using VCT and testing HIV-positive seemed more likely to lose a spouse but to acquire a regular non-cohabiting partner by Sero5, compared to those not using VCT. Conversely between Seros 5-6, those testing HIV-positive seemed more likely to acquire a spouse but to lose a regular partner by Sero6. These results may have arisen simply as a result of the small sample sizes which yielded nonstatistically significant results, but other factors could also have been at play. For example, antiretroviral therapy was more widely available in the study area between Sero5 and Sero6, and this may have assisted participants in regaining health and acquiring more permanent spousal partners. Sero-sorting could also have been taking place, with more people aware of their HIV-status by the later sero-survey rounds, allowing them to seek out other HIV-positive individuals with whom to form more permanent partnerships.

The limitations of this study relate to the observational nature of the data and to the pitfalls of working with self-reported sexual behaviour data, which are subject to recall and social desirability biases. We have previously shown that in Kisesa, VCT tends to attract individuals with higher risk sexual behaviours [19], and so as VCT uptake increases, the associations between VCT use and reductions in sexual risk behaviour among the general population may be weaker. In addition, we may have overstated the impacts of VCT if those who used the service were more likely to report declines in their risk behaviour compared to those who didn't. However, the community in Kisesa has been exposed to various HIV information and education campaigns over a number of years, and so all study participants are likely to have had some degree of exposure to HIV prevention messages.

Our ability to detect changes in levels of risk behaviour was limited by lack of knowledge of the sexual risk profile and HIV-status of partners. Take for example an individual who reported one sexual partner at both rounds, but who had an HIV-negative partner at the earlier round and an HIV-positive one at the later round. In this scenario, there would have been an increase in the level of risk between rounds which our behaviour change index would have been incapable of detecting. Further analyses among the subset of married and co-habiting couples for whom partner HIVstatus are available may warrant investigation.

The strengths of this study relate to the fact that our VCT use data were documented rather than self-reported, and we were able to adjust for reported use of HIV counselling and testing services outside of sero-surveys, which should have helped to reduce levels of measurement error and residual confounding.

\section{Conclusions}

We found moderate but encouraging associations between VCT use and reductions in some sexual risk behaviours among HIV-negative participants, including reductions in the number of sexual partners in the last year, and a move to safer types of partnership. However, the 
overall uptake of VCT was low and we found no impacts of VCT on changes in HIV incidence. There were also no significant or consistent impacts of VCT on changes in sexual behaviour among HIV-positive individuals, but small sample sizes may have prevented us from discovering these. The impact of VCT services on sexual behaviour change and HIV prevention will ultimately depend upon rates of uptake, with further research required to better understand processes of behaviour change following VCT use.

\section{Abbreviations}

VCT: Voluntary counselling and testing; ART: Antiretroviral therapy; PITC: Provider initiated testing and counselling; RR: Risk ratio; aRR: adjusted risk ratio; Cl: Confidence interval.

\section{Competing interests}

The authors declare that they have no competing interests.

\section{Authors' contributions}

CC developed the data analysis protocols, carried out the analyses and wrote the manuscript. AW, ES and BZ advised on data analysis protocols and amendments to these. JT, DM, MU, YK and BZ contributed to the conception, design and acquisition of the data. All authors critically reviewed the manuscript for important intellectual content. All authors read and approved the final manuscript.

\section{Acknowledgements}

We are grateful to the study participants and to the staff of the Kisesa cohort study. The Kisesa cohort study is funded by the Global Fund to Fight AIDS, Tuberculosis and Malaria. Data management activities have also been supported by a grant from the Wellcome Trust. The first author (Caoimhe Cawley) is funded by the UK Economic and Social Research Council.

\section{Author details}

'Department of Population Health, London School of Hygiene and Tropical Medicine, Keppel Street, London WC1E 7HT, UK. ${ }^{2}$ TAZAMA Project, National Institute for Medical Research, P. O. Box 1462, Mwanza, Tanzania.

Received: 18 September 2013 Accepted: 18 March 2014

Published: 22 March 2014

\section{References}

1. De Cock KM, Marum E, Mbori-Ngacha D: A serostatus-based approach to HIV/AIDS prevention and care in Africa. Lancet 2003, 362(9398):1847-1849.

2. Scaling-up HIV testing and counselling services: a toolkit for programme managers. [http://www.who.int/hiv/pub/vct/toolkit/en/index.html]

3. Denison JA, O'Reilly KR, Schmid GP, Kennedy CE, Sweat MD: HIV voluntary counseling and testing and behavioral risk reduction in developing countries: a meta-analysis, 1990-2005. Aids Behav 2008, 12(3):363-373.

4. The Voluntary HIV-1 Counseling and Testing Efficacy Study Group: Efficacy of voluntary HIV-1 counselling and testing in individuals and couples in Kenya, Tanzania, and Trinidad: a randomised trial. Lancet 2000, 356(9224):103-112.

5. Fonner VA, Denison J, Kennedy CE, O'Reilly K, Sweat M: Voluntary counseling and testing (VCT) for changing HIV-related risk behavior in developing countries. Cochrane Database Syst Rev 2012, 9:Cd001224.

6. Matovu JKB, Gray RH, Makumbi F, Wawer MJ, Serwadda D, Kigozi G, Sewankambo NK, Nalugoda F: Voluntary HIV counseling and testing acceptance, sexual risk behavior and HIV incidence in Rakai, Uganda. Aids 2005, 19(5):503-511.

7. Turner AN, Miller WC, Padian NS, Kaufman JS, Behets FM, Chipato T, Mmiro FA, Salata RA, Morrison CS: Unprotected sex following HIV testing among women in Uganda and Zimbabwe: short- and long-term comparisons with pre-test behaviour. Int J Epidemiol 2009, 38(4):997-1007.

8. Sherr L, Lopman B, Kakowa M, Dube S, Chawira G, Nyamukapa C Oberzaucher N, Cremin I, Gregson S: Voluntary counselling and testing: uptake, impact on sexual behaviour, and HIV incidence in a rural Zimbabwean cohort. Aids 2007, 21(7):851-860.
9. Dare 00 , Cleland JG: Reliability and validity of survey data on sexual behaviour. Health Transit Rev 1994, 4(Suppl):93-110.

10. Slaymaker $\mathrm{E}: \mathrm{A}$ critique of international indicators of sexual risk behaviour. Sex Transm Infect 2004, 80(Suppl 2):ii13-21.

11. Ferry B, Carael M, Buve A, Auvert B, Laourou M, Kanhonou L, de Loenzien M, Akam E, Chege J, Kaona F: Comparison of key parameters of sexual behaviour in four African urban populations with different levels of HIV infection. Aids 2001, 15(Suppl 4):S41-S50.

12. Boerma JT, Gregson S, Nyamukapa C, Urassa M: Understanding the uneven spread of HIV within Africa: comparative study of biologic, behavioral, and contextual factors in rural populations in Tanzania and Zimbabwe. Sex Transm Dis 2003, 30(10):779-787.

13. Buve A, Carael M, Hayes RJ, Auvert B, Ferry B, Robinson NJ, Anagonou S, Kanhonou L, Laourou M, Abega S, Akam E, Zekang L, Chege J, Kahindo M, Rutenberg N, Kaona F, Musonda R, Sukwa T, Morison L, Weiss HA, Laga M, Study Group on Heterogeneity of HIV Epidemics in African Cities: The multicentre study on factors determining the differential spread of HIV in four African cities: summary and conclusions. Aids 2001, 15(Suppl 4):S127-S131.

14. Cleland J, Boerma JT, Carael M, Weir SS: Monitoring sexual behaviour in general populations: a synthesis of lessons of the past decade. Sex Transm Infect 2004, 80(Suppl 2):ii1-ii7.

15. Mwaluko G, Urassa M, Isingo R, Zaba B, Boerma JT: Trends in HIV and sexual behaviour in a longitudinal study in a rural population in Tanzania, 1994-2000. Aids 2003, 17(18):2645-2651.

16. Wambura M, Urassa M, Isingo R, Ndege M, Marston M, Slaymaker E, Mngara J, Changalucha J, Boerma TJ, Zaba B: HIV prevalence and incidence in rural Tanzania: results from 10 years of follow-up in an open-cohort study. J Acquir Immune Defic Syndr 2007, 46(5):616-623.

17. Urassa M, Isingo R, Marston M, Kumugola Y, Mngara J, Changalucha J, Jonas A, Mtenga B, Beckles D, Zaba B: Direct evidence of recent change in HIV prevalence and incidence in a rural population cohort in Northern Tanzania, 1994-2007. In XVIII International AIDS Conference: 2010; Vienna, Austria. 2010.

18. Ministry of Health Tanzania: National Guidelines for Voluntary Counselling and Testing, 2005. Edited by National AIDS Control Programme. Tanzania; 2005.

19. Isingo R, Wringe A, Todd J, Urassa M, Mbata D, Maiseli G, Manyalla R, Changalucha J, Mngara J, Mwinuka E, Zaba B: Trends in the uptake of voluntary counselling and testing for HIV in rural Tanzania in the context of the scale up of antiretroviral therapy. Trop Med Int Health 2012, 17(8):e15-e25.

20. Wringe A, Floyd S, Kazooba P, Mushati P, Baisley K, Urassa M, Molesworth A, Schumacher C, Todd J, Zaba B: Antiretroviral therapy uptake and coverage in four HIV community cohort studies in sub-Saharan Africa. Trop Med Int Health 2012, 17(8):e38-e48

21. Corbett EL, Makamure B, Cheung YB, Dauya E, Matambo R, Bandason T, Munyati SS, Mason PR, Butterworth AE, Hayes RJ: HIV incidence during a cluster-randomized trial of two strategies providing voluntary counselling and testing at the workplace, Zimbabwe. Aids 2007, 21(4):483-489.

22. Mola OD, Mercer MA, Asghar RJ, Gimbel-Sherr KH, Gimbel-Sherr S, Micek MA, Gloyd SS: Condom use after voluntary counselling and testing in central Mozambique. Trop Med Int Health 2006, 11(2):176-181.

23. Plummer ML, Wight D, Wamoyi J, Mshana G, Hayes RJ, Ross DA: Farming with your hoe in a sack: condom attitudes, access, and use in rural Tanzania. Stud Fam Plann 2006, 37(1):29-40.

24. Mwanga JR, Mshana G, Kaatano G, Changalucha J: "Half plate of rice to a male casual sexual partner, full plate belongs to the husband": findings from a qualitative study on sexual behaviour in relation to HIV and AIDS in northern Tanzania. BMC Public Health 2011, 11:957.

\section{doi:10.1186/1471-2334-14-159}

Cite this article as: Cawley et al:: The impact of voluntary counselling and testing services on sexual behaviour change and HIV incidence: observations from a cohort study in rural Tanzania. BMC Infectious Diseases 2014 14:159. 